\title{
Behavior-based Control Considering the Interaction Between a Human Operator and an Autonomous Surface Vehicle
}

\author{
Yonghoon Cho $\circledast^{*}$, Jonghwi Kim $\circledast^{*}$, Jinwhan Kim $\circledast^{*}$, Yongjin Jo $\circledast^{* *}$ and Jaekwan Ryu $\circledast^{* *}$ \\ "Department of mechanical engineering, KAIST, Daejon, Korea \\ *Unmanned/Robotic systems, LIG Nex1 CO., Ltd., Pangyo, Korea

\section{운용자와 자율 무인선 상호 작용을 고려한 행위 기반의 제어 알고리즘} \\ 조용훈(ํㅗㅁ \\ "한국과학기술원 기계공학과 \\ "LIG넥스원 미래기술연구소
}

KEY WORDS: Behavior based control 행위기반제어, Unmanned surface vehicles 무인선, Robot system architecture, 로봇 시스템 구조, Artificial intelligence 인공지능, Human operator 운용자

\begin{abstract}
With the development of robot technology, the expectation of autonomous mission operations has increased, and the research on robot control architectures and mission planners has continued. A scalable and robust control architecture is required for unmanned surface vehicles (USVs) to perform a variety of tasks, such as surveillance, reconnaissance, and search and rescue operations, in unstructured and time-varying maritime environments. In this paper, we propose a robot control architecture along with a new utility function that can be extended to various applications for USV. Also, an additional structure is proposed to reflect the operator's command and improve the performance of the autonomous mission. The proposed architecture was developed using a robot operating system (ROS), and the performance and feasibility of the architecture were verified through simulations.
\end{abstract}

\section{1. 서 론}

최근 로봇이 운용되는 임무 및 환경이 복잡해지고 요구되는 기능 수준이 향상됨에 따라 복잡한 상황에 대응하기 위한 로봇 시스템 구조(Robot system architecture)와 함께 이를 구현 가능한 소프트웨어 도구의 필요성이 증가하고 있다(Kortenkamp el al., 2016). 특히. 끊임없이 변화하는 비정형화된 환경을 인지하고 이에 대해 대응하는 기능이 필수적으로 요구되는 무인 이동체 (Unmanned vehicles)는, 공장자동화 시스템과 같이 반복된 작업 을 수행하는 로봇과 달리, 복잡하게 구성된 체계(System)를 세 분화 된 하위체계(Sub-system)로 분리하여 체계화 할 필요성이 더욱 높다(Siciliano and Khatib, 2016). 이러한 필요성에 맞춰 Brooks(1986)은 기존의 기능(function) 중심의 시스템을 행위 (Behavior) 중심의 시스템으로 변경하여 다수 임무, 다수 센서, 강건성, 확장성에 강점이 있는 행위 기반 로봇(Behavior-based robot)을 제안한 바 있다.
무인선의 경우 감시, 정찰, 수색, 인명구조 등의 다양한 임무 를 여러 종류의 센서를 활용하여 수행하되 임무 환경인 해양 환경에서의 강건한 성능을 확보하기 위해 행위 로봇 구조의 활 용이 시도되었다. 대표적인 연구 사례들로, Benjamin et al. (2006)은 Mission oriented operating suite(MOOS) 기반의 행위 로 봇 구조를 설계하고 무인선에 적용하였으며, 미 제트추진연구 소(JPL, Jet Propulsion Laboratory)는 행위 로봇 기반의 Control architecture for robotic agent command and sensing (CARACAS)을 무인선에 적용하여 자율운항 및 협업구조를 실해역에서 테스트 하였다(Elkins et al., 2010; Wolf el al., 2017).

기존 연구의 경우 무인선을 포함하는 무인 이동체 내의 인공 지능을 최대로 구현하는 연구에 중점을 두고 있으나, 실제적인 무인선 운용은 임무 수행 간 원격 운용자의 개입 및 감시가 필 수적으로 요구된다. 따라서, 무인선이 만족스러운 임무 수행 능 력을 보이지 않거나 특수 상황에서의 필요성에 따라 운용자의 의도를 반영한 의사 결정이 이루어져야 하겠으나, 기존 시스템

Received 11 October 2019, revised 1 November 2019, accepted 23 November 2019

Corresponding author Jinwhan Kim: +82-42-350-1519, jinwhan@kaist.ac.kr ORCID: http://orcid.org/0000-0001-6886-2449

(c) 2019, The Korean Society of Ocean Engineers

This is an open access article distributed under the terms of the creative commons attribution non-commercial license (http://creativecommons.org/licenses/by-nc/3.0) which permits unrestricted non-commercial use, distribution, and reproduction in any medium, provided the original work is properly cited. 
의 경우 임무 수행 시 원격 운용자와 무인선과의 상호 작용을 통한 행동 선택(Action selection)에 대한 고려 없이 운용자가 모 든 절차를 전적으로 제어하는 원격 조작(Remote operation)의 초 보적 개념에 치우쳐 있는 것이 현실이다.

따라서, 본 연구에서는 무인선의 임무 수행 시 자율 운항 핵 심 기능을 유지하되 원격 운용자의 의도를 함께 고려하여 의사 결정이 가능한 운용자와 자율 알고리즘의 상호 협력적 무인선 로봇 시스템 구조를 제안한다. 우선, 운용자의 개입 없이 무인 선의 자율임무 수행이 가능하도록 행위 기반의 로봇 시스템 구 조를 설계하고, 원격 운용자의 명령 시 해당 명령을 고려하기 위한 구조를 설계하고 추가하였다. 여러 행위가 복합적으로 행 해질 때, 최종 목표 행동을 계산하기 위하여 행동 공간(Action space) 내에서의 행동 평균(Action averaging)방법을 활용한 행동 선택 방법을 사용하였으며, 이를 무인선에 적용하기 위해 효용 함수(Utility function)를 설계하였다. 설계된 구조의 유용성을 확 인하기 위하여, 소프트웨어 도구로서 Robot operating system (ROS)을 활용하였으며(Koenig and Howard, 2004), 시뮬레이션을 활용하여 해당 방법의 유용성을 검증하였다.

\section{2. 행위 기반 로봇}

운용자의 개입 없이 무인선의 자율임무 수행을 위한 기본 행 위 기반 구조를 Fig. 1과 같이 설계하였다. 구조는 입력순서에 따라 행위 관리 도구(Behavior manager), 행위의 병렬적 집합, 최 적화 도구(Optimizer)의 세 가지 주 요소로 구성되어 있다. 센싱/ 인지 시스템은 행위 기반 구조에 무인선이 임무를 수행하기에 충분하도록 발생한 위치와 속도를 포함하는 운동 정보와 해당 정보의 유형을 제공한다고 가정하였으며, 이를 이벤트(Event)로 정의하였다. 행위 관리 도구는 센싱/인지 시스템에서 수신한 이 벤트 정보를 바탕으로 하여 실행 중인 행위의 관리를 담당하며, 각 행위는 행위에 알맞은 행동 공간에서의 효용 함수를 생성하 고, 최적화 도구는 행동 공간 내에서 최적의 행동을 찾는 역할 을 담당한다.

운용자가 무인 이동체에 명령(Command)을 내리기 위해서는 운용자의 명령을 로봇이 이해 가능하도록 변형하여 로봇이 반 영할 수 있게 해야 한다. 행위 기반 로봇의 경우 이를 다른 구 조에 비해 쉽게 구현할 수 있다는 장점이 존재하며, 무인 이동 체의 자율임무 수행 시 운용자의 개입에 의한 구조는 Fig. 2 와 같다. 기존 무인 이동체의 판단 구조에 운용자의 명령을 반영할 수 있는 구조를 추가했으며, 명령 관리 도구와 각 명령의 집합

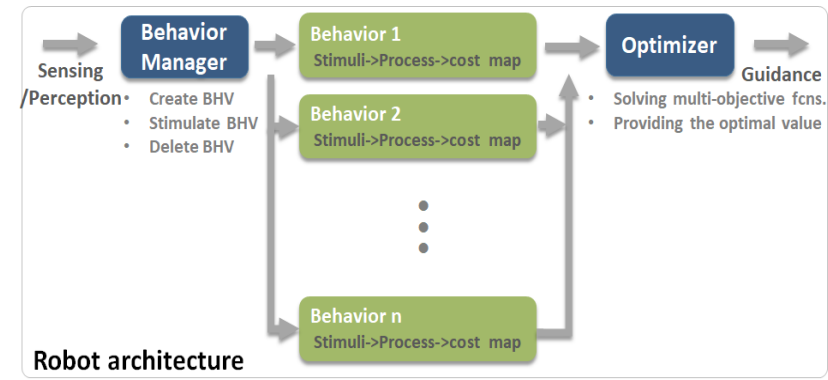

Fig. 1 Behavior based architecture without a human operator.

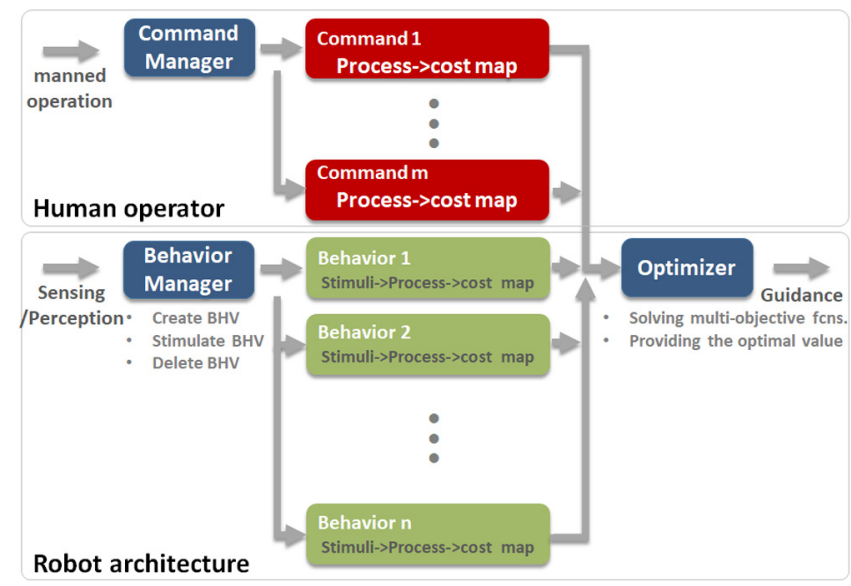

Fig. 2 Behavior based architecture with a human operator; Green boxes represent the behavior and red boxes represent the command from a human operator.

으로 이루어져 있다. 무인 이동체의 행동 관리 도구 및 행위와 명령 관리 도구 및 명령의 집합은 각 요소끼리 대응적인 관계 를 갖고 유사한 기능을 수행하며, 명령은 무인 이동체가 이해할 수 있는 행위의 일종으로 표현되어 운용자의 명령을 무인 이동 체가 자신의 판단 하에서 수행할 수 있도록 한다. 최적화 도구 에서는 기존의 행위만을 고려하던 기능에서 추가된 명령에서의 효용 함수를 추가적으로 고려하여 최적의 행동을 찾도록 한다. 하지만. 기존 무인 이동체의 행위 판단 구조와는 다르게 센싱 /인지 시스템이 따로 존재하지 않고, 사람이 인지 정보를 통하 여 판단으로 얻어낸 명령을 입력으로 사용하며, 명령을 관리하 기 위한 명령 관리 도구는 운용자가 내린 모든 명령을 수행할 수 있도록 동작하고, 명령은 행위보다 상위 개념으로서 행위보 다 우선적으로 수행하도록 한다.

\section{1 행위 관리 도구 및 명령 관리 도구의 설계}

행위 관리 도구는 센싱/인지 시스템에서 수신한 이벤트 정보를 바탕으로 하여 행위의 생성(Create)/자극(Stimulate)/제거(Delete)를 담당한다. 행위 관리 도구 내 행위의 생성 기능은 무인선의 센서 /인지 시스템에서 수신받은 이벤트에 적합한 행위를 생성하는 역할을 수행하며, 이벤트가 다수 발생하는 상황에서도 이에 해 당하는 적합한 다수 행위를 생성하도록 설계되었다. 센싱/인지 시스템으로부터 전달된 이벤트와 이에 대응하는 행위의 조합은 일대일 혹은 필요 시 다대다로 조합되도록 구성되었다.

행위의 자극 기능은 수행된 행위 중 수행이 요구되는 이벤트 에 대하여 행위를 자극하여 적합한 업무를 수행할 수 있도록 하며, 자극으로 인해 임무를 수행 중인 행위의 상태를 활성화 (Activation) 상태로 정의하고, 임무를 수행 중이지 않은 행위는 비활성화(Deactivation) 상태로 정의하여 무인선이 수행해야 하 는 행위를 분류하였다. 행위의 자극은 생성된 모든 행위 중 일 정 반경 이내에 존재하는 이벤트에 대하여 실시하며, 일정 반경 밖의 이벤트는 비활성화되도록 설계하였다.

행위의 제거 기능은 수행 완료된 이벤트를 다시 수행하지 않 기 위하여 해당 행위를 제거하는 기능을 수행하며, 수행 완료된 이벤트에 대하여 해당하는 다수의 행위를 동시에 제거하거나, 
혹은 이벤트에 대한 일부 행위를 제거하는 역할을 수행하도록 설계하였다.

명령 관리 도구는 행위 관리 도구와 유사한 기능을 수행하지 만, 운용자의 명령 전달 시 이미 명령이 생성된 상태로 간주하 고 명령의 생성 시 자극을 동시에 수행하며, 명령 내린 행위가 완료된 것으로 판단 시 명령을 제거하도록 설계되었다.

\section{2 행동 선택 알고리즘(Action selection mechanism)}

행동 선택 알고리즘은 그 종류에 따라 크게, 경쟁적(Competitive) 구조와 협력적(Cooperative) 구조로 구분할 수 있으며(Arkin, 1998), 행위가 동시에 수행될 때 경쟁적 구조는 하나의 행위 혹은 행동 을 선택하나 협력적 구조는 여러 개의 행위를 조합하여 하나의 새로운 행동을 제시한다. 운용자의 명령을 포함하는 순차적이지 않은 다수의 행동을 동시에 수행하기 위해서는 하나의 행동을 선택하는 구조는 실제적으로 비효율적이므로, 협력적 구조의 일 환인 행동 공간 내에서 최선의 행동을 선택하는 방법을 선택하 였다.

행동 공간은 현재 운동상태에서 목표로 하는 운동상태의 집 합이며, 목표 속력과 목표 침로의 순서쌍인 아래의 식으로 나타 낼 수 있다.

$$
A=\left\{\left\langle\psi_{i}, V_{i}\right\rangle|| V_{i}-V\left|<\gamma_{V}, V>0,\right| \psi_{i}-\psi \mid<\gamma_{\psi}\right\}
$$

여기서, $V_{i}$ 와 $\psi_{i}$ 는 목표 속력과 목표 침로의 행동 공간 집합의 요소를 의미하며, $V$ 와 $\psi$ 는 현재 운동체의 속력과 침로를 의미 한다. 현재 속력과 침로에서 도달 가능한 목표 속력과 침로를 행동 공간으로 정의하기 위하여 속력과 침로 변화량의 문턱값 $\gamma_{V}$ 와 $\gamma_{\psi}$ 를 사용하였다.

각 행동 공간 내에서 효용 함수를 $f_{k}$ 로 정의 시 효용 함수는 행위의 함수로 표현이 가능하며, 최적의 행동은 아래의 식으로 나타낼 수 있다.

$$
\langle\hat{\psi}, \hat{V}\rangle=\underset{\left\langle\psi_{i}, V_{i}\right\rangle \in A}{\arg \max }\left[\sum_{k=1}^{n} w_{k} f_{k}\left(\psi_{i}, V_{i}\right)\right]
$$

여기서, $\langle\hat{\psi}, \hat{V}\rangle$ 는 최적 행동의 순서쌍을 의미하며, $w_{k}$ 는 $k$ 번 째 행위의 가중치를 의미한다. 무인 이동체가 행해야 하는 우선 순위가 높은 행위일수록 높은 가중치를 갖는다.

운용자의 명령을 반영하기 위하여, 행위의 효용 함수에 명령 으로 인해 생긴 효용 함수를 추가적으로 고려한다. 명령은 행위 와 같은 행동 공간 내에서 행위와 함께 반영되나, 그 우선순위 는 행위에 비하여 우선순위가 높으며, 명령이 반영된 최적의 행 동은 아래의 식으로 나타낼 수 있다.

$$
\langle\hat{\psi}, \widehat{V}\rangle=\underset{\left\langle\psi_{i}, V_{i}\right\rangle \in A}{\arg \max }\left[\sum_{k=1}^{n} w_{k} f_{k}\left(\psi_{i}, V_{i}\right)+\sum_{k=1}^{m} w_{k}^{c} f_{k}^{c}\left(\psi_{i}, V_{i}\right)\right]
$$

여기서, $w_{k}^{c}$ 는 $\mathrm{k}$ 번째 명령의 가중치를 의미하고, $f_{k}^{c}$ 는 $\mathrm{k}$ 번째 명 령의 효용 함수를 나타낸다. 명령 또한 행위와 마찬가지로 명령 의 종류에 따라 그 우선순위가 달라지거나, 혹은 운용자의 운용
에 따라 그 우선순위가 달라지므로 가중치를 통하여 명령 수행 순위를 조절한다.

\section{3. 무인선을 위한 행위 기반 구조의 적용}

무인선의 자율 임무 수행 시 운용자와의 상호 작용을 위하여 행위기반구조 내 적절한 행위 및 임무의 설계가 필요하다. 무인 선이 수행 가능한 임무는 기본적으로 익수자 구출, 의심 선박 추적, 고정 장애물 및 이동 장애물의 회피로 설정하였으며, 행 위 기반 로봇의 특성상 무인선의 추가 임무 수행 필요 시 설계 및 추가가 가능하다.

또한, 운용자와의 상호 작용을 위해서 운용자가 무인선에게 전달 가능한 명령의 설계가 요구되며, 해당 명령은 회피와 이동 으로 설정하였다. 앞선 행위 기반 로봇의 특성과 동일하게 회피 와 이동 이외에 운용자가 내릴 수 있는 추가 명령이 필요 시 설 계 및 추가가 용이하다.

\section{1 무인선 행위의 설계}

행위는 전달받은 이벤트에 따라 익수자와 추종 선박은 이동 (Go)행위, 고정 장애물은 회피(Avoid)행위, 회피선박은 회피행위 와 규정 준수(Rule-compliant) 행위를 동시에 수행하는 반응 (Reactive)구조로 설계되었으며, 전역경로를 추종하는 행위는 이 동 행위의 연속으로 설계하였다. 이동 행위는 자선의 속도 좌표 계로 이루어진 행동 공간 내에서 추종제어(Pursuit guidance)를 이용하여 이벤트의 발생 지점으로 이동하는 목적함수를 생성하 며, 회피 행위는 행동 공간 내에서 최근접 거리(DCPA, Distance to the closest point of approach)를 기반으로 목적함수를 생성하 였다. 행동 공간 내에서 이동 행위와 회피 행위의 효용함수 $f^{g o}$ 와 $f^{a v}$ 는 각각 식 (3)과 식 (4)와 같다.

$$
\begin{aligned}
f_{i}^{g o}= & 1-\frac{\alpha_{\psi}\left|\beta-\psi_{i}\right|+\alpha_{V}\left|V-V_{i}\right|}{\max \left(\alpha_{\psi}\left|\beta-\psi_{i}\right|+\alpha_{V}\left|V-V_{i}\right|\right)} \\
f_{i}^{a v} & = \begin{cases}0 & \text { if } D C P A_{i}<\gamma_{D C P A}^{\min }, \\
1 & \text { if } D C P A_{i}>\gamma_{D C P A}^{\max }, \\
\frac{0.9\left(D C P A_{i}-\gamma_{D C P A}\right)}{\left(\gamma_{D C P A}^{\max }-\gamma_{D C P A}^{\min }\right)} & \text { oth erwise }\end{cases}
\end{aligned}
$$

여기서 $\psi_{i}$ 와 $V_{i}$ 는 행동 공간 내 자선의 행동을 의미하고, $\alpha_{\psi}$ 와 $\alpha_{V}$ 는 행동 공간의 속도와 방위각의 가중치를 의미한다. $D C P A_{i}$ 는 순서쌍 $\left\langle\widehat{\psi}_{i}, \widehat{V}_{i}\right\rangle$ 에서의 최근접 거리를 의미하며, $\gamma_{D C P A}^{\min }$ 와 $\max _{D C P A}$ 는 회피 행위를 생성하기 위한 최근접거리의 하계(Lower bound) 문턱값과 상계(Higher bound) 문턱값을 의미한다. 규정준 수 행위는 장애물의 조우 시 국제해상충돌예방규칙(COLREGs, International regulations for preventing collisions at sea)을 생성하 도록 구성되며(Kuwata et al., 2013), 회피 행위와 복합적으로 구 성된다. 경로 추종 행위는 임무 시작 전 설정되거나, 임무 도중 자동으로 생성된 일련의 경유점을 추종하는 역할을 수행하며, 이동 행위의 순차적 집합으로 구현되어 있다. 목표 경유점에 도 착 시 다음 경유점으로 변경하는 경유점 변경 알고리즘(Waypoint 


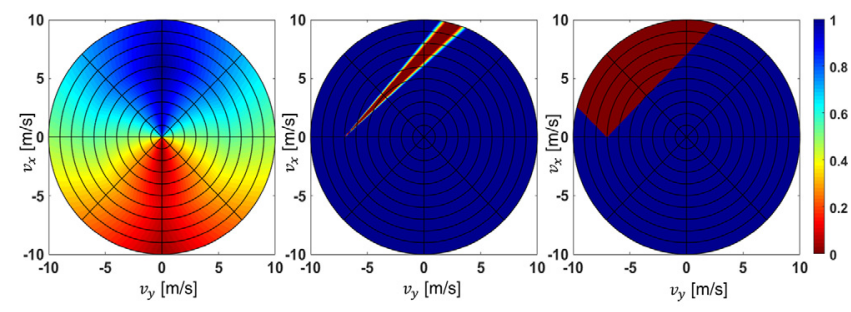

Fig. 3 Examples of behavior cost in the action space; go behavior (left); avoid behavior (center); rule-compliant behavior (right).

shifting algorithm)이 존재하여 이동 행위를 통한 순차적인 경유 점 추종을 가능하게 한다. 각 행위의 행동 공간 내 효용 함수의 예시는 Fig. 3과 같다.

\section{2 무인선 명령의 설계}

무인선과의 상호 작용을 위한 명령의 설계는 이동과 회피로 설계하였다. 운용자가 내리는 명령은 그 명령의 목적보다는 명 령에 대응되는 행위가 중요하므로, 명령에 따른 행위가 직관적 으로 대응되도록 설계하였으며, 이동 명령은 이동 행위를 생성 및 자극하며, 회피 명령은 회피 행위를 생성 및 자극하도록 설 계 되었다. 이동 명령과 회피 명령에 대한 효용 함수는 각 명령 에 대응되는 식 (3)과 식 (4)와 같으며, Fig. 3의 왼쪽 및 중앙과 동일하다.

\section{4. 시뮬레이션 결과}

제안하는 구조의 유용성을 검증하기 위하여, Fig. 4 및 Table 1 과 같은 시뮬레이션 환경에서 무인선의 자율임무 수행을 확인 하였다.

시뮬레이션은 미리 설정된 전역경로와 고정장애물 3 대, 이동 장애물 1 척, 무인선이 구출해야 하는 조난자 2 명으로 구성되어 있다. 무인선은 행위 기반 구조에 기반하여 각 상황에 맞는 행 동을 계산하며, 운용자가 중간에 내리는 명령을 수행하며 자동 임무 수행을 하도록 한다. 시률레이션 시 각 임무의 탐지 및 인 식 거리는 $300 \mathrm{~m}$ 이며, 임무의 완료는 $20 \mathrm{~m}$ 이내로 임무에 접근할 경우로 가정하였다. 각 행위를 실행하기 위한 행위의 가중치는 Table 2와 같다.

Table 1 Simulation environment

\begin{tabular}{ccccc}
\hline \hline \multirow{2}{*}{ Event } & \multicolumn{5}{c}{ State } \\
\cline { 2 - 5 } & $x[\mathrm{~m}]$ & $y[\mathrm{~m}]$ & Heading $\left[{ }^{\circ}\right]$ & Speed $[\mathrm{m} / \mathrm{s}]$ \\
\hline Buoy1 & 300.0 & -250.0 & - & - \\
Buoy2 & -200.0 & 100.0 & - & - \\
Buoy3 & 200.0 & 100.0 & - & - \\
MO1* & 50.0 & -50.0 & 135.0 & 2.0 \\
Person1 & -300.0 & 100.0 & - & - \\
Person2 & 200.0 & 200.0 & - & - \\
waypoint & 400.0 & -400.0 & - & - \\
\hline
\end{tabular}

*MO: Moving obstacle

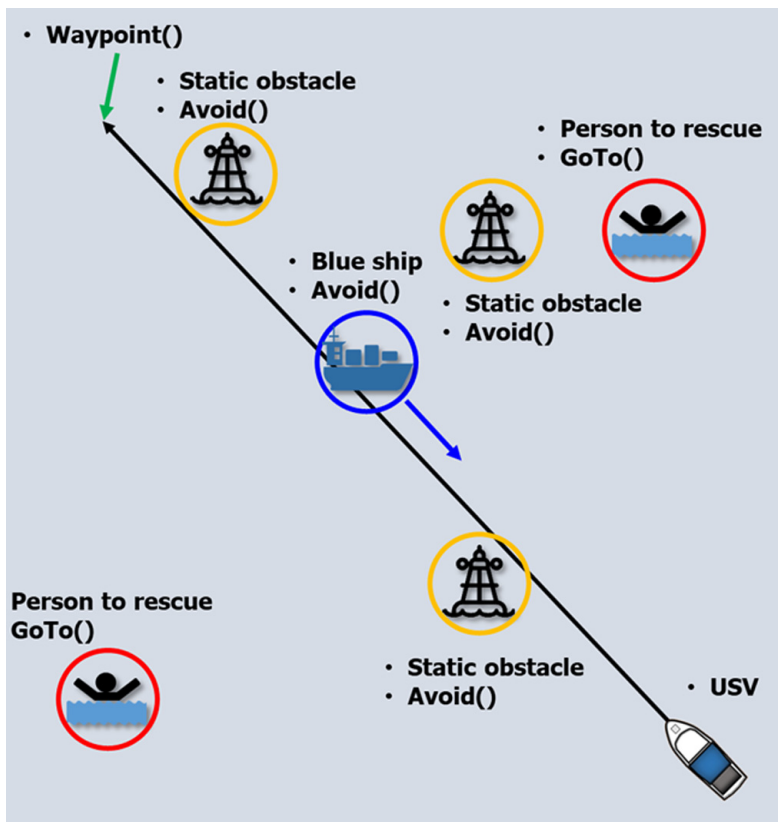

Fig. 4 Initial simulation environment with person to rescue, static obstacles and moving obstacles.

Table 2 Weighting parameter $\left(w_{k}, w_{k}^{c}\right)$

\begin{tabular}{ccc}
\hline \hline \multirow{4}{*}{ Event } & Parameter $\left(w_{k}, w_{k}^{c}\right)$ \\
\hline \multirow{3}{*}{ Event } & Waypoint & 0.5 \\
& Rescue & 2.0 \\
& Static obstacle & 5.0 \\
& Moving obstacle & 5.0 \\
\hline \multirow{2}{*}{ Command } & Avoid & 10.0 \\
& Go & 1.0 \\
\hline
\end{tabular}

행위의 가중치는 가중치의 절대치 보다는 행위의 우선순위인 상대값이 큰 의미를 가지며, 유사한 이벤트에 대하여 운용자의 명령이 무인선의 행위에 비하여 높은 우선순위를 갖기에 더 높 은 가중치를 부여하였다. 또한, 장애물회피와 같이 무인선 안전 을 위협하는 상황에 대한 가중치는 명령 및 행위에 상관없이 높은 가중치를 가지며, 상대적으로 임무를 수행하거나 이동하 는 명령 및 행위는 낮은 가중치를 갖도록 설계하였다.

\section{1 시뮬레이션 결과}

Fig. 5-7은 각각 사용자 명령이 존재하지 않는 경우, 이동 명 령이 존재하는 경우, 회피 명령이 존재하는 경우의 주요 시간별 결과이다. 각 결과 그래프의 좌측은 무인선의 시간별 임무 수행 을 묘사하며, 우측은 그 순간의 무인선의 속도 좌표계에서의 행 동 공간 내 효용 함수를 나타낸다. 임무 수행 묘사 그림에서 흑 색 실선은 무인선의 전역 경로이며 흑색 파선은 무인선의 이동 경로이다. 청색 오각형은 무인선을, 황색 오각형은 이동 장애물 을 나타내며, 적색, 녹색, 주황색, 자색 원은 각각 고정 장애물, 익수자, 이동 명령, 회피 명령을 나타낸다. 또한, 회색 실선은 현재 활성화된 이벤트를 나타낸다. 

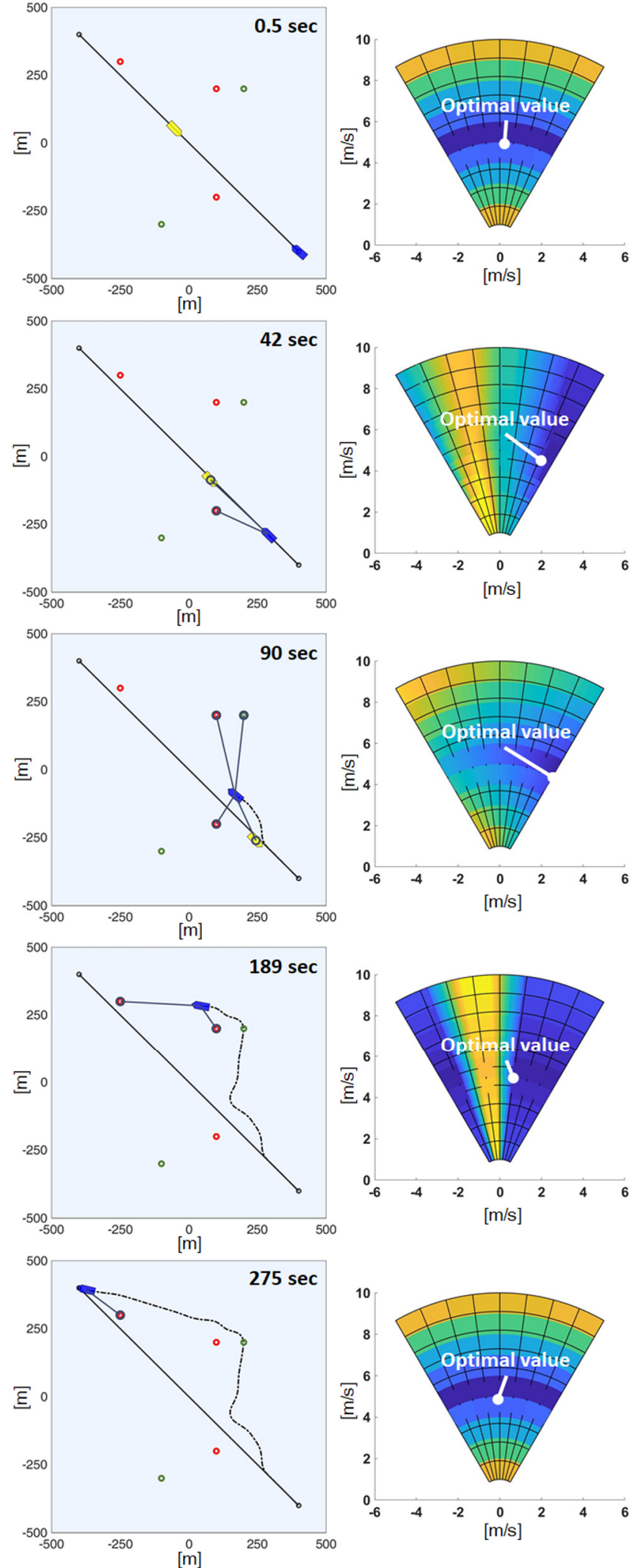

Fig. 5 Simulation result without user command; trajectory and mission (left); utility function in action space (right).

Fig. 5의 사용자의 명령이 존재하지 않는 경우에는 주어진 전 역 경로를 추종하며, 무인선이 처한 상황에 따라 고정 및 이동 장애물을 회피하고, 익수자를 구출하며 최종 경유점에 도착한
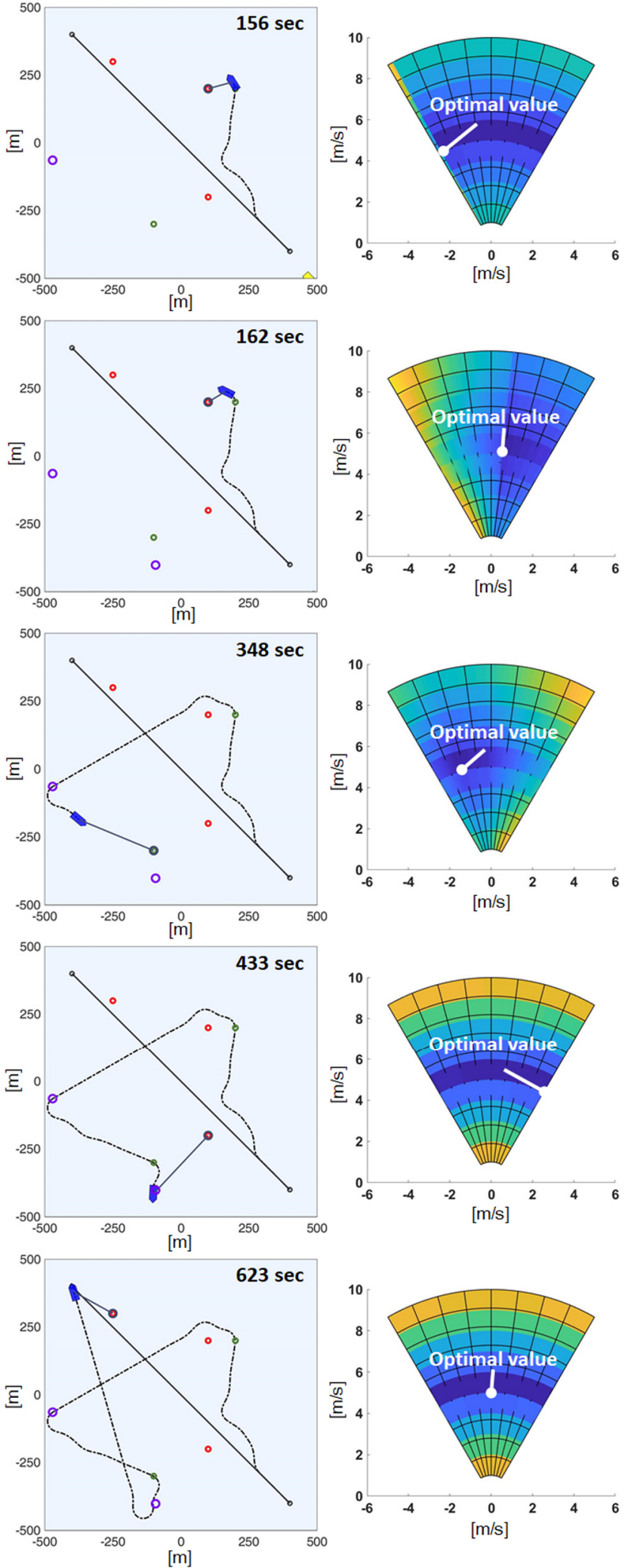

Fig. 6 Simulation result with go command; trajectory and mission (left); utility function in action space (right).

다. Fig. 6의 이동 명령이 존재하는 경우는, Fig. 5와 같은 시나 리오에서 사용자가 이동 명령을 내리는 156 초 이전까지는 같은 임무 수행 결과를 도시하나, 156 초와 162 초에 이동 명령 수신 
Behavior-based Control Considering the Interaction Between a Human Operator and an Autonomous Surface Vehicle 625
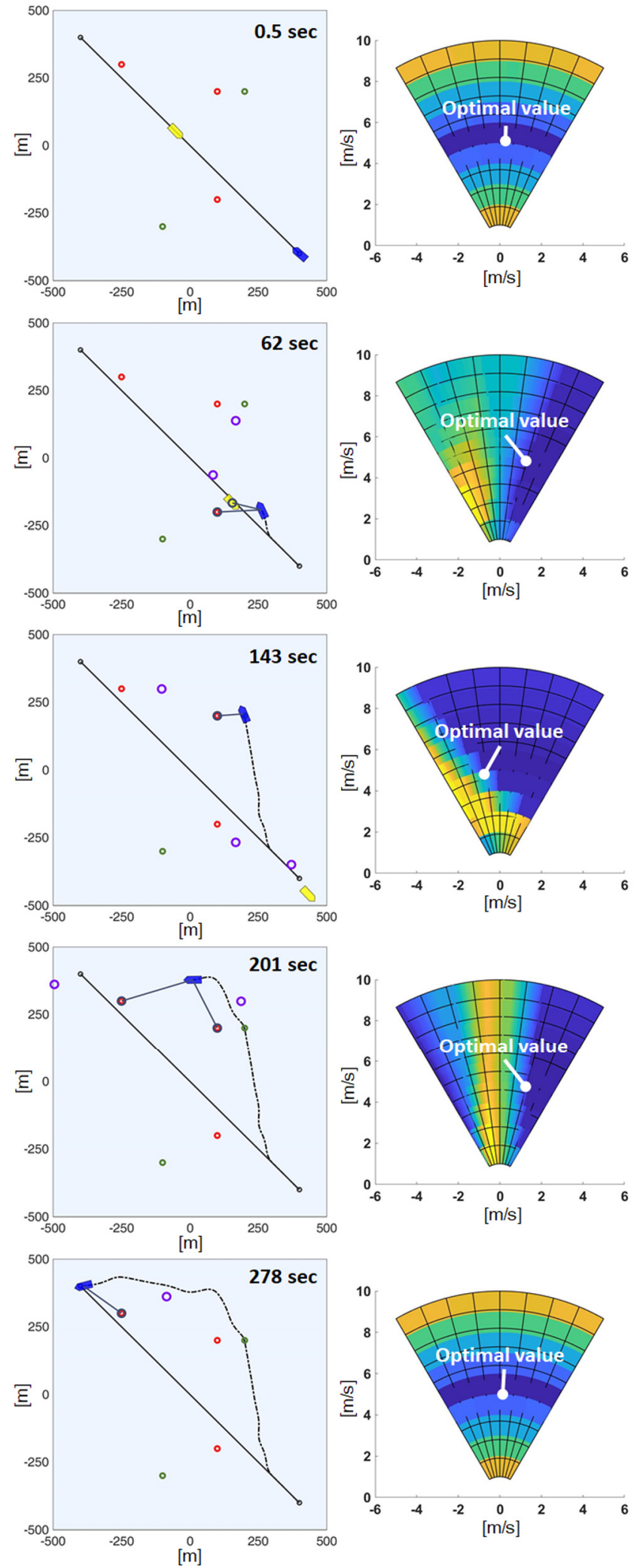

Fig. 7 Simulation result with avoid command; trajectory and mission (left); utility function in action space (right).

후 이동 명령을 수행하며, 단순히 사용자의 이동 명령만을 수행 하는 것이 아닌 이동 명령 중 나타나는 상황에 적절히 대응하 여 첫 번째 이동 명령 완료 후 익수자를 구출하기 위한 행위를
시도하는 것을 확인 가능하다.

Fig. 7의 사용자의 회피 명령이 존재하는 경우 해당 이동 장 애물에 대하여 적절한 회피 기작을 수행하는 것을 확인 가능하 며, 사용자의 명령뿐만 아니라 자율 임무 수행 중 탐지/인식된 익수자에 대하여 구출 행위를 하거나 고정장애물을 회피 하는 것을 확인 가능하다.

\section{5. 결 론}

본 논문에서는 무인선의 자율 임무 수행을 위한 행위 기반 구 조 및 운용자와의 상호 작용을 위한 구조 설계에 대하여 다루 었다. 무인선의 자율 임무 수행 간 마주치는 상황을 익수자 구 출 및 장애물 조우로 정의하고 해당 상황의 해결을 위한 효용 함수를 설계하였다. 또한, 운용자의 명령을 이동과 회피 행위로 정의하고 해당 명령을 행위로 변경하여 무인선이 운용자의 명 령을 적절히 반영할 수 있도록 하였다. 설계된 행위 및 명령과 이를 활용하기 위한 행위 기반 구조를 검증하기 위하여 시뮬레 이션을 진행하였으며, 시뮬레이션 결과를 정성적으로 평가 시 운용자의 명령과 행위 기반 구조 기반의 무인선이 상황에 따라 적절한 행동을 선택함을 확인할 수 있다.

시뮬레이션을 통하여 설계한 구조가 복잡한 환경에서 적절한 행동을 선택함을 정성적으로 확인하였으나, 해당 방법은 반응 구조를 기반으로 설계하였기 때문에, 실제적인 운용은 가능하 나 최적성을 보장하기는 어렵다. 따라서, 설계된 구조 내에서 자율 임무를 수행함에 있어 최적성을 보장할 절차가 필요할 것 으로 생각되며, 향후 연구로서 반응구조와 심의구조를 적절히 융합하여 최적성을 보장하는 기법 개발이 바람직할 것으로 판 단되고, 시뮬레이션 환경 기반 검증을 실험기반의 검증으로 확 장하여, 제안하는 알고리즘의 실제적 검증이 필요할 것으로 생 각된다.

\section{후기}

본 연구는 LIG 넥스원의 '무인수상정의 자율 운용 알고리즘 프레임워크 구축 및 적용 연구'의 지원으로 수행된 연구결과 중 일부임을 밝히며, 연구비 지원에 감사드립니다.

\section{References}

Arkin, R.C., 1998. Behavior-based Robotics. MTT Press.

Benjamin, M.R., Leonard, J.J., Curcio, J.A., Newman, P.M., 2006. A Method for Protocol-based Collision Avoidance Between Autonomous Marine Surface Craft. Journal of Field Robotics, 23(5), 333-346. https://doi.org/10.1002/rob.20121

Brooks, R., 1986, A Robust Layered Control System for a Mobile Robot. IEEE Journal on Robotics and Automation, 2(1), 14-23. https://doi.org/10.1109/JRA.1986.1087032

Elkins, L., Sellers, D., Monach, W.R., 2010. The Autonomous Maritime Navigation (AMN) Project: Field Tests, Autonomous and Cooperative Behaviors, Data Fusion, Sensors, and Vehicles. Journal 
of Field Robotics, 27(6), 790-818. https://doi.org/10.1002/rob.20367 Koenig, N., Howard, A., 2004. Design and Use Paradigms for Gazebo, an Open-source Multi-robot Simulator. Proceedings of IEEE/RSJ International Conference on Intelligent Robots and Systems (IROS), 3, 2149-2154. https://doi.org/10.1109/IROS.2004.1389727

Kortenkamp, D., Simmons, R., Brugali, D., 2016. Robotic Systems Architectures and Programming. Springer Handbook of Robotics, 2nd Edition, Springer, Cham.

Kuwata, Y., Wolf, M.T., Zarzhitsky, D., Huntsberger, T.L., 2013. Safe Maritime Autonomous Navigation with COLREGS, Using Velocity Obstacles. IEEE Journal of Oceanic Engineering, 39(1), 110-119.
https://doi.org/10.1109/JOE.2013.2254214 Siciliano, B., Khatib, O. (Eds.), 2016. Springer Handbook of Robotics. Springer. https://doi.org/10.1007/978-3-540-30301-5

Wolf, M.T., Rahmani, A., de la Croix, J.P., Woodward, G., Vander Hook, J., Brown, D., Schaffer, S., Lim, C., Bailey, P., Tepsuporn, S., Pomerantz, M., Nguyen, V., Sorice, C., Sandoval, M., 2017. CARACaS Multi-agent Maritime Autonomy for Unmanned Surface Vehicles in the Swarm II Harbor Patrol Demonstration. Proceedings of Unmanned Systems Technology XIX, International Society for Optics and Photonics, 101950O. https://doi.org/10.1117/12.2262067 